\title{
A NEW OUTBURST IN THE EXTRAORDINARY CENTRAL STAR OF LMC-N66 $6^{1,2,3}$
}

\author{
Miriam Peña, ${ }^{4}$ Maria Teresa Ruiz, ${ }^{5}$ Patricio Rojo, ${ }^{5}$ Silvia Torres-Peimbert,${ }^{4}$ and Wolf-Rainer Hamann ${ }^{6}$
}

\begin{abstract}
This is the first report on the new outburst presented by the central star of the LMC-N66 nebula. This object was classified as a planetary nebula; however, its true nature is under debate. In the period 1955-1990 the central star was almost undetectable and only nebular emission lines were observed. In 1990, the beginning of an outburst was detected and in few months it became much brighter and developed wide $\mathrm{He}$ and $\mathrm{N}$ lines, typical of a WolfRayet star of the N-sequence. The maximum occurred in 1994 and afterward the star slowly faded. Analysis of its evolution showed that it has a variable mass-loss rate which occasionally increases enormously, creating a false photosphere at a much larger radius, making it appear a few magnitudes brighter. The present outburst has occurred 13 years after the episode from 1994 to 2000. So far this new event has similar characteristics although there are some significant differences in the spectral features. We present optical and FUSE spectra showing the main properties of this latter event.
\end{abstract}

Subject headings: planetary nebulae: individual (LMC-N66) — stars: mass loss — stars: winds, outflows — stars: Wolf-Rayet

Online material: color figure

\section{INTRODUCTION}

In 1993, a huge mass-loss event was detected in the central star of the planetary nebula LMC-N66 (also SMP 83 and WS 35). The star developed features of a Wolf-Rayet star (WR) of the $\mathrm{N}$-sequence and its brightness increased by a large factor while the nebular characteristics remained unchanged (Peña et al. 1994, 1995). The event was monitored at UV and optical wavelengths. The mass loss gradually decreased and the star returned to its quiet state in about 6 years. A detailed analysis of the stellar evolution, using advanced non-LTE models for expanding atmospheres fitting the UV and optical data across these epochs, was performed by Hamann et al. (2003), who showed that the stellar luminosity increased from $\log \left(L / L_{\odot}\right)=4.6$ at the end of 1990 (quiescence) to 5.4 in 1994 and then returned gradually to the initial value. In the same period the mass-loss rate increased from (quiescence) $10^{-5.7}$ to $10^{-5.0} M_{\odot} \mathrm{yr}^{-1}$ at maximum, while the stellar temperature remained constant during the whole event $\left(T_{*}=\right.$ $112 \pm 20 \mathrm{kK})$. The chemical composition of the ejecta corresponded to that of an incompletely $\mathrm{CNO}$-processed material: dominated by helium $\left(X_{\mathrm{He}}=0.8\right)$ with a small amount of hydrogen $\left(X_{\mathrm{H}}=0.2\right)$, nitrogen slightly enhanced $\left(X_{\mathrm{N}}=3 \times\right.$ $\left.10^{-3}\right)$, and carbon very depleted $\left(X_{\mathrm{C}} \leq 1 \times 10^{-4}\right)$. Several possible scenarios for the nature of LMC-N66 (single massive star, post-AGB star, high-mass binary system with mass transfer, or white dwarf accreting mass) are discussed by Hamann et al. Although none of the scenarios are completely satisfactory, it is suggested that the most probable ones are those involving an evolved binary system. In particular, LMC-N66 could be a

\footnotetext{
${ }^{1}$ ESO proposals ID 279.D-5024 and ID 076.B-0166.

${ }^{2}$ Based on observations made with the NASA-CNES-CSA Far Ultraviolet Spectroscopic Explorer (FUSE), operated for NASA by the John Hopkins University under NASA contract NAS5-32985.

${ }^{3}$ Based on observations obtained at Las Campanas (Carnegie)

${ }^{4}$ Instituto de Astronomía, Universidad Nacional Autónoma de México, Apartado Postal 70264, México, D.F. 04510, México; miriam@astroscu.unam.mx.

${ }^{5}$ Departamento de Astronomía, Universidad de Chile, Casilla 36D, Santiago, Chile.

${ }^{6}$ Universität Potsdam, Am Neuen Palais 10, 14469 Potsdam, Germany.
}

white dwarf accreting matter at a high rate in a close binary. The large accretion may bring the stellar mass to the Chandrasekhar limit within a few hundred thousand years, making it a candidate for a Type Ia supernova precursor.

Due to its extraordinary characteristics we have kept monitoring this object at irregular intervals over the years. The last spectrum obtained that showed no outburst was taken on 2006 January 28. Fifteen months later, on 2007 April 26, a new outburst was discovered.

\section{OBSERVATIONS}

Once the outburst was detected, a follow-up program was started right away, thanks to a Director's Discretionary Time (DDT) program, with EMMI and the NTT at European Southern Observatory (ESO). Concurrently we obtained DDT with the FUSE satellite. In this section we describe these observations.

\subsection{Optical Spectrum}

A spectrum obtained with the ESO VLT and FORS1 spectrograph on 2006 January 28 shows the star in quiescence, presenting a faint continuum and faint $\mathrm{He}$ II 4686 wide component. The outburst was discovered in 2007 April 26 with an optical spectrum of N66 obtained at Las Campanas using the duPont telescope equipped with the B\&C spectrograph. Right after the discovery, ESO DDT was granted through program ID 279.D-5024. The ESO-NTT + EMMI spectrograph with a combination of gratings has been used to gather spectroscopic data in the 3250-8000 Å wavelength range. Spectra were obtained on 2007 July 22, August 14, August 30, September 14, and October 22. The exposure times were of 20 minutes in the blue and 10 minutes in the red.

The spectrum from 2007 August 30 is shown in Figure 1 together with the data obtained on 2006 January 28 (quiescence) and a spectrum from 1994 August 2 (maximum of previous outburst). Some stellar characteristics, derived from these spectra, are presented in Table 1. It is evident that during outbursts the star increases its brightness and develops wide and prominent $\mathrm{WN}$ features (mainly $\mathrm{He}$ II, $\mathrm{N} \mathrm{v}$, and $\mathrm{N}$ IV lines). 


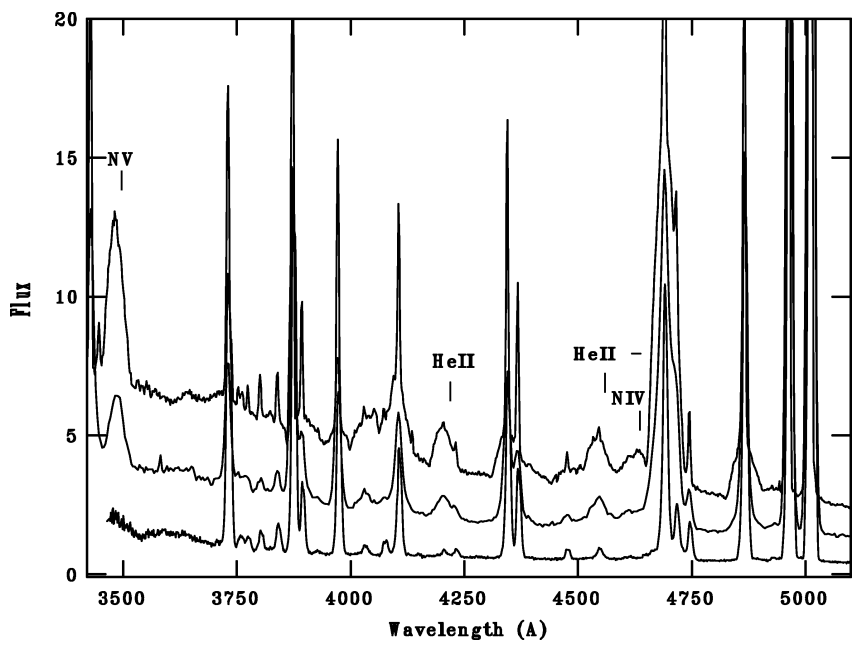

FIG. 1.-Optical spectra of LMC-N66 for 2007 August 30 (top), 1994 (middle), and 2006 (bottom) are presented, showing the new outburst, the previous one, and the quiescent state. Flux is in units of $10^{-15} \mathrm{erg} \mathrm{cm}^{-2} \mathrm{~s}^{-1} \AA^{-1}$. The most intense WR features are marked. The 2007 spectrum shows a brighter stellar continuum and more intense WR features probably due to a larger massloss rate.

For the two observed outbursts the spectra are very similar, presenting the same WN features, although the stellar magnitude in 2007, as measured from the continuum flux at 5450 $\AA$, is brighter than in 1994 by $0.5 \mathrm{mag}$ and about $2.2 \mathrm{mag}$ brighter than in quiescence. Note that due to the differences in slit widths, the flux listed for 1994 and 2006 corresponds to the whole stellar flux, while in the 2007 observation some of the emission could have been lost due to seeing conditions. The mass-loss rate listed in Table 1 for 1994 was taken from Hamann et al. (2003), and for 2006 we adopted the quiescent value derived by Hamann et al.; alternatively, for 2007 we estimated $\dot{M}$ by scaling the value of 1994, taking into account that $F(\mathrm{He}$ II 4686) is 1.7 times more intense in 2007 and that $\dot{M} \propto \sqrt{F}$.

In 2007 the stellar continuum is brighter and also the WR emission lines are more intense, which could be understood in terms of a higher mass-loss rate. On the other hand, the WR features present very similar FWHMs in both outbursts, indicating similar wind velocities. There are other small differences between outbursts, for instance the N v $3484 \AA$ line is stronger in 2007 than in 1994, and the C IV 5808 doublet is more prominent, relative to $\mathrm{He}$ II $5411 \AA$ and $\mathrm{He}$ I $5876 \AA$, as can be observed in Figure 2, where we present the 5300-6000 zone normalized to the continuum. It is known that the stellar spectrum of LMC-N66 undergoes small variations on short timescales (of about a month); therefore it seems likely that the observed differences in the spectra between 2007 and 1994

TABLE 1

Stellar Characteristics for Different Epochs

Parameter

Slit width $(\operatorname{arcsec}) \ldots \ldots \ldots \ldots$

$F(\mathrm{He} \text { II } 4686)^{\mathrm{a}}$

FWHM(He II 4686) $(\AA)$

Stellar continuum ${ }^{\mathrm{b}} \ldots \ldots \ldots \ldots$

$m_{V} \ldots \ldots \ldots \ldots \ldots \ldots \ldots \ldots$

$\log \dot{M} \ldots \ldots \ldots \ldots \ldots \ldots \ldots$

${ }^{a}$ Flux in units of erg $\mathrm{cm}^{-2} \mathrm{~s}^{-1}$.

${ }^{\mathrm{b}}$ Stellar continuum at $5450 \AA$, in units of $\mathrm{erg} \mathrm{cm}^{-2} \mathrm{~s}^{-1} \AA^{-1}$. The nebular contribution has been subtracted.

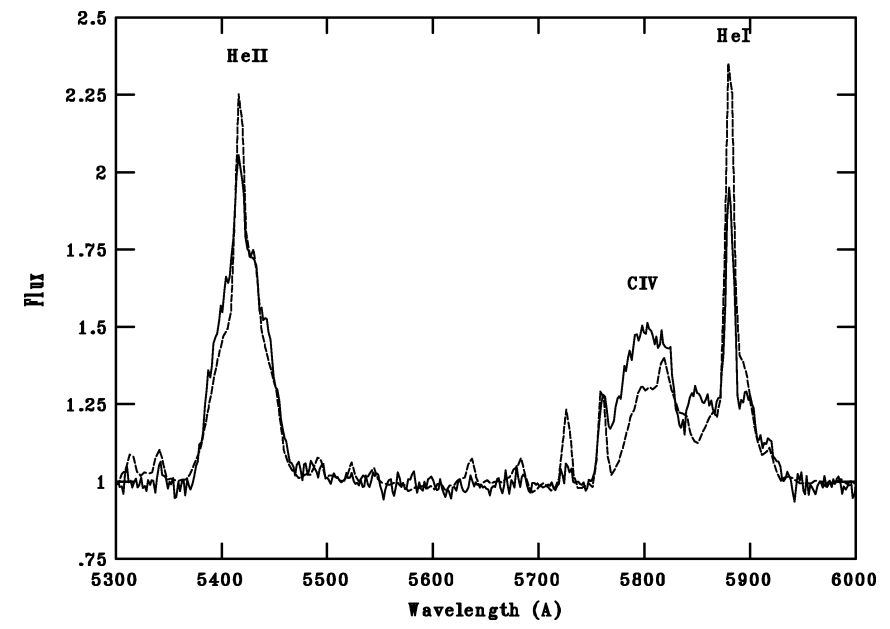

FIG. 2.-Comparison of He II 5411, He I 5876, and C IV 5808 features for 2007 (solid line) and 1994 (dashed line) observations. The spectra have been normalized to compare the wide emission features. The $\mathrm{C}$ IV 5808 line is much brighter, relative to the $\mathrm{He}$ lines, in the 2007 event.

could be due to short-time variations of the wind parameters and do not necessarily reflect differences in chemical composition or other important parameters.

\subsection{FUV Spectrum}

Due to the high stellar temperature, the maximum emission from the star is at short wavelengths; therefore it was very important to secure UV information. FUSE DDT was granted through the program ID Z018. The observations covered the wavelength range 905-1187 $\AA$, at a spectral resolution of $R \sim$ 20,000 . Originally an exposure time of $27,000 \mathrm{~s}$ was approved, but unfortunately the termination of the FUSE science mission in 2007 August made it impossible to complete the full exposure. Only two observations (ID Z0180101000 of $8235 \mathrm{~s}$ and Z0180102000 of 5783 s) were acquired in 2007 July. Nevertheless the stellar brightness was sufficient to obtain data with significant signal-to-noise ratios. The FUSE LWRS $\left(30^{\prime \prime} \times\right.$ $\left.30^{\prime \prime}\right)$ aperture was used. The data, taken in "tag time" mode, were calibrated using the FUSE data reduction pipeline.

A combination of the signal obtained through the four channels (LiF1, LiF2, SiC1, SiC2) of the two detectors (A and B) is presented in Figure 3. This spectrum was smoothed to reduce the spectral resolution to $0.2 \AA$. Wide emission lines from the

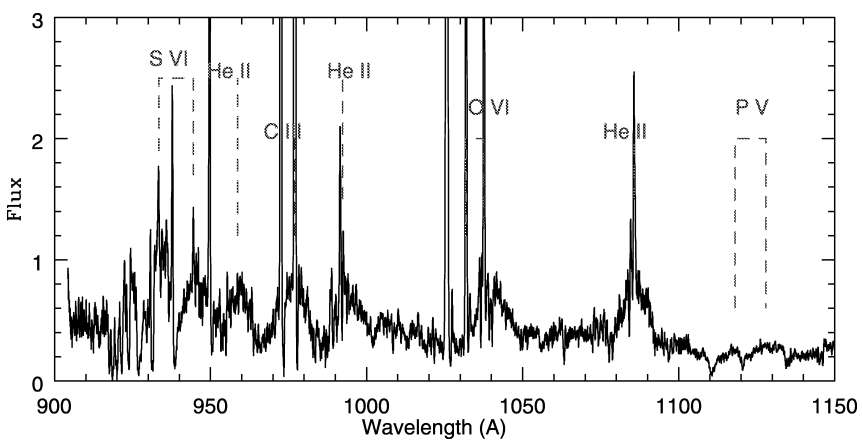

FIG. 3.-FUSE spectrum of the central star of LMC-N66 in 2007 July. Flux in units of $10^{-13} \mathrm{erg} \mathrm{cm}^{-2} \mathrm{~s}^{-1} \AA^{-1}$. The marked WR features are similar to the ones of a WN 3 star (see Willis et al. 2004). Narrow nebular lines of the Lyman series (produced by airglow) and nebular C III (977 $\AA$ ), He II (992 $\AA), \mathrm{O}$ VI $(1032,1038 \AA)$, and He II (1086 $\AA$ ) are also very intense. [See the electronic edition of the Journal for a color version of this figure.] 
central star are clearly appreciated. The most prominent lines correspond to He II, S VI, O VI, and P V. According to Willis et al. (2004) these features correspond to a high-excitation WN (see their Figs. $2 a$ and $2 b$ and their $\S 4.2$ ). The most important stellar lines and their characteristics are listed in Table 2. Previous FUSE data for LMC-N66 central star were published by Herald \& Bianchi (2007). In comparison with their spectrum, obtained in 2003 at quiescent state (see their Fig. 1), our 2007 spectrum shows a continuum 5 times more intense and welldeveloped WR lines.

In addition to the wide stellar lines, narrow emission lines from the Lyman series are conspicuous. These lines correspond to the airglow, but some nebular lines such as C III $977 \AA$, O VI 1032 , $1038 \AA$, and He II $1086 \AA$ are observed. Narrow absorptions displaced $1 \AA$ to the red from the Lyman airglow lines are produced by interstellar absorption in the LMC ( $1 \AA$ corresponds to the radial velocity of $300 \mathrm{~km} \mathrm{~s}^{-1}$ measured for LMC-N66 by Peña et al. 2004).

\section{DISCUSSION}

By comparing the characteristics of the far UV stellar lines with those of the FUSE spectra for massive WR stars published by Willis et al. (2004), we find that the spectral classification for the central star of LMC-N66 corresponds to an early WN, probably a WN 3. In the previous outburst, and based on optical spectra only, we classified it as a WN 4.5. Certainly this object is not a classical WR star as it is much less luminous, it presents outbursts difficult to understand, and in addition, its spectrum shows short-time variability that yields a slightly different spectral classification. For 2007, the optical spectrum shows N IV and $\mathrm{He}$ I lines, so the WN 4.5 classification is still valid. But, as said above, the spectral classification for this object is not as direct as in the case of massive WRs due to its peculiarities.

Undoubtedly the central star of LMC-N66 is undergoing a new huge mass-loss event only 13 years after the previous episode. In this event, the star appears about 0.5 mag brighter than in the previous one and shows more intense WR features. A period as short as $13 \mathrm{yr}$ for a new outburst was not expected. Although the star was reported by Nail \& Shapley (1955) as a variable (HV 5967) with $\Delta m \sim 0.9$ mag, subsequent variations were not reported for more than 30 years, although this object was observed at least in 1975, 1976, 1983, 1985, 1988, 1989, and 1990 by different authors (e.g., Dopita et al. 1985;
TABLE 2

Far UV Stellar WR Emission Features

$\lambda_{0}$

\begin{tabular}{|c|c|c|c|}
\hline$(\AA)$ & Ion & Flux $^{\mathrm{a}}$ & Comments \\
\hline 933.4 & S VI & 6.54 & P Cygni \\
\hline 944.5 & S VI & 6.46 & P Cygni \\
\hline 958.7 & $\mathrm{He}$ II & 3.41 & Blended with $\mathrm{N}$ IV? \\
\hline 977.0 & C III & 5.29 & Nebular C III \\
\hline $992.3 \ldots \ldots \ldots$ & $\mathrm{He}$ II & 3.72 & Nebular He II \\
\hline $1031.8+37.5 \ldots \ldots$ & $\mathrm{O}$ VI & 4.38 & Nebular O vI \\
\hline $1085.7 \ldots \ldots \ldots \ldots$ & $\mathrm{He}$ II & 3.74 & Nebular He II \\
\hline 1118.0 . & $\mathrm{P} v$ & 0.50 & P Cygni \\
\hline $1128.0 \ldots$ & $\mathrm{P} v$ & 1.10 & P Cygni \\
\hline
\end{tabular}

Monk et al. 1988; Peña \& Ruiz 1988; Meatheringham \& Dopita 1991; Dopita et al. 1993). None of these observations were made in a year corresponding to a probable maximum in an apparent $13 \mathrm{yr}$ period (going back in time, the years for a maximum would have been 1981, 1968, 1955). But taking into account that the 1994 outburst declined slowly and that the star was a couple of magnitudes brighter than in quiescence and the stellar lines were prominent for a few years after maximum, such features would have been detected had they been present in other epochs. Therefore there is no evidence for an outburst of the intensity of the ones in 1994 and 2007 to have taken place from 1955 to 1990.

Thus there is no evidence for periodicity in the stellar variations. The LMC-N66 central star can only be classified as an irregular variable with a mass-loss that can increase enormously occasionally and we cannot predict the next outburst. We continue to monitor the star in the optical range, to follow its evolution, and to verify whether the spectral differences between 2004 and 1994 persist (see $\S 2.1$ and Table 1). The data obtained will be used to compute new atmosphere models to detect any variation in the stellar parameters.

M. Peña is grateful to DAS, Universidad de Chile, for hospitality during a sabbatical stay when part of this work was performed. This work received financial support from DGAPAUNAM (IN-118405) and CONACyT (46904). M. T. Ruiz and P. Rojo acknowledge support from the FONDAP Center for Astrophysics and from the Centro de Astrofísica y Tecnologías Asociadas (CATA).

\section{REFERENCES}

Dopita, M. A., Ford, H. C., Bohlin, R., Evans, I. N., \& Meatheringham, S. J. 1993, ApJ, 418, 804

Dopita, M. A., Ford, H. C., Lawrence, C. J., \& Webster, B. L. 1985, ApJ, 296, 390

Hamann, W.-R., Peña, M., Gräfener, G., \& Ruiz, M. T. 2003, A\&A, 409, 969

Herald, J. E., \& Bianchi, L. 2007, ApJ, 661, 845

Meatheringham, S. J., \& Dopita, M. A. 1991, ApJS, 75, 407

Monk, D. J., Barlow, M. J., \& Clegg, R. E. S. 1988, MNRAS, 234, 583

Nail, V. M., \& Shapley, H. 1955, Proc. Nat. Acad. Sci., 41, 685
Peña, M., Hamann, W.-R., Ruiz, M. T., Peimbert, A., \& Peimbert, M. 2004, A\&A, 419, 583

Peña, M., Peimbert, M., Torres-Peimbert, S., Ruiz, M. T., \& Maza, J. 1995, ApJ, 441, 343

Peña, M., \& Ruiz, M. T. 1988, RevMexAA, 16, 55

Peña, M., Torres-Peimbert, S., Peimbert, M., Ruiz, M. T., \& Maza, J. 1994, ApJ, 428, L9

Willis, A. J., et al. 2004, ApJS, 154, 651 